\title{
Observation of gravitational waves by light polarization
}

\author{
Chan Park ${ }^{1, \mathrm{a}}\left(\mathbb{D}\right.$, Dong-Hoon Kim ${ }^{2, \mathrm{~b}}$ \\ ${ }^{1}$ National Institute for Mathematical Sciences, Daejeon 34047, Republic of Korea \\ 2 Department of Physics and Astronomy, Seoul National University, Seoul 08826, Republic of Korea
}

Received: 10 December 2020 / Accepted: 18 January 2021 / Published online: 30 January 2021

(C) The Author(s) 2021

\begin{abstract}
We provide analysis to determine the effects of gravitational waves on electromagnetic waves, using perturbation theory in general relativity. Our analysis is performed in a completely covariant manner without invoking any coordinates. For a given observer, using the geometrical-optics approach, we work out the perturbations of the phase, amplitude, frequency and polarization properties-axes of ellipse and ellipticity of light, due to gravitational waves. With regard to the observation of gravitational waves, we discuss the measurement of Stokes parameters, through which the antenna patterns are presented to show the detectability of the gravitational wave signals.
\end{abstract}

\section{Introduction}

Light serves as a powerful means to observe gravitational waves. In fact, most of the current and future gravitationalwave detectors make use of light. As a primary example, laser interferometers observe gravitational waves through the interference patterns of light caused by the difference of the photon transit times between two arms [1-3]. Viewing this from a different perspective, the electromagnetic waves propagating along each arm are perturbed by gravitational waves, and the observation of gravitational waves is enabled by the difference between the phases of the perturbed electromagnetic waves from the two arms: the electromagnetic waves interfere when they meet at the intersection of the arms, where a trace of the gravitational wave signals would cause changes to the intensity of the superposed electromagnetic waves [4]. As another example, pulsar timing arrays observe gravitational waves through the changes in the electromagnetic pulse periods from pulsars. The phase of electromagnetic waves is delayed during the passage of gravitational waves, which eventually causes the measured pulse

\footnotetext{
${ }^{a}$ e-mail: iamparkchan@gmail.com

b e-mail: ki13130@gmail.com (corresponding author)
}

frequency (or period) to vary slightly. Then the cumulative variation of this (termed as a residual) enables the observation of gravitational waves [5]: taking account of the crosscorrelation of the residuals of two pulsars nearby in the sky (i.e., the quadrupolar interpulsar correlation) would enhance confirmation of the observation [6].

There have been numerous studies investigating the effects of gravitational waves on light in the context of general relativity. Some of the studies focus on the effects on the polarization of light. Among others, Montanari [7] obtained a solution of Maxwell equations in a gravitational wave background to one order of approximation beyond the geometrical-optics limit, and applied this to the study of perturbations of the linear polarization of electromagnetic waves. Calura and Montanari [8] presented the exact solution to the linearized Maxwell equations in spacetime slightly curved by a gravitational wave only in the framework of the linearized general relativity, without invoking the geometrical-optics approximation, and applied this to the case of a linearly polarized electromagnetic field bounced between two parallel conducting planes. Halilsoy and Gurtug [9] analyzed the Faraday rotation in the polarization vector of a linearly polarized electromagnetic shock wave upon encountering with gravitational waves. Hacyan $[10,11]$ determined the influence of a gravitational wave on the elliptic polarization of light, deducing the rotation of the polarization angle and the corresponding Stokes parameters, and applied this effect to the detection of gravitational waves, as a complement to the pulsar timing method. Cabral and Lobo [12] obtained electromagnetic field oscillations induced by gravitational waves and found that these lead to the presence of longitudinal modes and dynamical polarization patterns of electromagnetic radiation.

In this paper, we employ perturbation theory of general relativity to analyze the influence of gravitational waves on electromagnetic waves, concentrating mainly on the effects on the polarization of light. Our analysis is then applied to the observation of gravitational waves by means of Stokes parameters. Largely, the paper proceeds in three steps 
through Sects. 2-4. In Sect. 2, we review the basics of gravitational and electromagnetic waves as described in the flat spacetime background, and introduce our notational conventions used in the paper. In Sect. 3, we work out a perturbation of electromagnetic waves due to gravitational waves from the perturbed Maxwell equations: using the geometricaloptics approach, the perturbations of the phase, amplitude, frequency and polarization properties-axes of ellipse and ellipticity of light are determined. In Sect. 4, application of our analysis to the observation of gravitational waves is discussed. Stokes parameters are employed as optical observables to identify the gravitational wave signals from, and measured in a suitable observational frame. The antenna patterns are defined via the Stokes parameters to exhibit the detectability of the gravitational wave signals.

Throughout the paper, our analysis is conducted in a completely covariant manner without invoking any coordinates. To express tensors, Roman indices $(a, b, c, \ldots)$ are used; however, they should be distinguished from other unitalicized or Greek or parenthesized subscripts used occasionally for special notations; e.g., $M_{\mathrm{o}}, M_{\epsilon}, \omega_{\mathrm{g}}, \omega_{\mathrm{e}}, \mathcal{E}_{(p)}, \mathcal{E}_{(s)}$, etc. Also, a parenthesized number on the top left of the surrounding text denotes the degree of perturbation; e.g., ${ }^{(0)} T_{a b}$ for the unperturbed tensor $T_{a b},{ }^{(1)} T_{a b}$ for the first-order perturbation of $T_{a b}$, etc. We use the geometrized unit system $(c=1, G=1)$ for gravitation and the Gaussian unit system $\left(\epsilon_{0}=\frac{1}{4 \pi}, \mu_{0}=4 \pi\right)$ for electromagnetism.

\section{Preliminaries}

To discuss a perturbation of a quantity $X$ in a spacetime $\left(M_{\mathrm{O}},{ }^{(0)} \mathrm{g}\right)$, where $M_{\mathrm{O}}$ denotes a background manifold with a metric ${ }^{(0)} g$, we consider a one-parameter family of perturbed spacetimes $\left(M_{\epsilon}, g(\epsilon)\right)$, where $\epsilon$ is a perturbation parameter, and $M_{\epsilon}$ is a manifold associated with $\epsilon$, with a metric $g(\epsilon)$ defined on it, such that $\left(M_{\epsilon}, g(\epsilon)\right)$ tends to $\left(M_{\mathrm{o}},{ }^{(0)} g\right)$ as $\epsilon \rightarrow$ 0 . Then, a perturbation $\delta X$ of the quantity $X$ is defined as the pull-back of $X$ from $M_{\epsilon}$ to $M_{\mathrm{o}}$ through a map between the two manifolds, subtracted by $X$ on $M_{\mathrm{o}}$. By Taylor expansion, $\delta X$ can be split into pieces of orders of $\epsilon$; i.e., $\delta X={ }^{(1)} X+$ ${ }^{(2)} X+O\left(\epsilon^{3}\right)$, where ${ }^{(1)} X$ and ${ }^{(2)} X$ denote first-order and second-order perturbations, respectively.

A gauge transformation associated with a perturbation corresponds to changing a map between $M_{\mathrm{o}}$ and $M_{\epsilon}$. Then, a gauge transformation of a first-order perturbation is described by ${ }^{(1)} X^{\prime}-{ }^{(1)} X=\mathcal{L}_{\xi} X$, where ${ }^{(1)} X$ and ${ }^{(1)} X^{\prime}$ are first-order perturbations via two different maps, and $\mathcal{L}_{\xi} X$ denotes the Lie derivative of $X$ with respect to $\xi$, a vector of $O(\epsilon)$ defined from the two maps. A first-order perturbation (1) $X$ is guage-invariant if $\mathcal{L}_{\xi} X$ vanishes for all $\xi$ : it is possible only if $X$ is zero, or a constant scalar, or constructed by Kronecker delta with constant coefficients. This approach was first introduced in [13] and reviewed later in [14].

Let us consider the Minkowski spacetime as the background manifold $M_{0}$. Then, a first-order perturbation of the Riemann tensor is gauge-invariant as the Riemann tensor vanishes in the background spacetime. With the stress-energy tensor being assumed to vanish to first order in $\epsilon$, a firstorder perturbation of Einstein equations becomes the classical gravitational wave equations: $\partial^{c} \partial_{c} h_{a b}=0$, with gauge conditions $\partial^{b} h_{a b}=0$ and $h^{a}{ }_{a}=0$, where $h_{a b}$ denotes a first-order perturbation of a metric $g_{a b}$, and $\partial$ is the partial derivative associated with the metric ${ }^{(0)} g$ in the Minkowski spacetime. We consider a monochromatic plane wave solution: $h_{a b}=\mathcal{H}_{a b} e^{\mathrm{i} P}$, where $\mathcal{H}_{a b}$ is a complex constant amplitude, and $P$ is a real phase scalar that satisfies ${ }^{(0)} n^{a} \partial_{a} P<0$ for an arbitrary observer with the 4-velocity ${ }^{(0)} n^{a}$ in the Minkowski spacetime and vanishes at the second covariant derivative. Also, the propagation vector for gravitational waves is defined as $k^{a} \equiv \partial^{a} P$, which is a constant null vector.

For an inertial observer with ${ }^{(0)} n^{a}$ living in the Minkowski spacetime, the propagation vector $k^{a}$ can be decomposed into $k^{a}=\omega_{\mathrm{g}}\left({ }^{(0)} n^{a}+\kappa^{a}\right)$, where $\omega_{\mathrm{g}} \equiv-{ }^{(0)} n^{a} \partial_{a} P>0$ is the frequency of gravitational waves measured by the observer, and $\kappa^{a}$ is a spatial unit vector orthogonal to ${ }^{(0)} n^{a}$ in the observer's point of view. Further, if we impose an additional gauge condition $h_{a b}{ }^{(0)} n^{b}=0$ (which together with $h^{a}{ }_{a}=0$ constitutes a transverse-traceless gauge), then the "electric" part of the Riemann tensor perturbation becomes ${ }^{(1)} R_{a c b d}{ }^{(0)} n^{c(0)} n^{d}=\frac{1}{2} \omega_{\mathrm{g}}^{2} h_{a b}$ : it should be noted that $h_{a b}$ in our gauge conditions is proportional to a gauge-invariant perturbation ${ }^{(1)} R_{a c b d}{ }^{(0)} n^{c(0)} n^{d}$.

Although the observer's 4-velocity $n^{a}$ is not constant in a perturbed spacetime $M_{\epsilon}$, we can impose the geodesic condition $n^{b} \nabla_{b} n^{a}=0$, with $\nabla$ being the covariant derivative associated with the metric $g(\epsilon)$ in $M_{\epsilon}$, which reduces to ${ }^{(0)} n^{b} \partial_{b}{ }^{(1)} n^{a}=0$ in $M_{0}$; i.e., ${ }^{(1)} n^{a}$ can be set to a constant along ${ }^{(0)} n^{a}$. For instance, we can set ${ }^{(1)} n^{a}=0$, which implies that the 4-velocity of the geodesic observer in $M_{\epsilon}$ is not perturbed by gravitational waves to first-order; namely, $\delta n^{a}=O\left(\epsilon^{2}\right)$. Throughout our analysis, we set ${ }^{(1)} n^{a}=0$, and $\delta n^{a}=0$ to first order in $\epsilon$.

Taking account of Einstein-Maxwell equations, we assume no electric charge for a source. Through the equations, the influence of the electromagnetic field on the spacetime geometry is of second order, and a first-order perturbation of the equations becomes the same classical gravitational wave equations as above.

In the Lorenz gauge $\partial^{a(0)} A_{a}=0$, Maxwell equations become the classical electromagnetic wave equations: $\partial^{b} \partial_{b}{ }^{(0)} A_{a}=0$, where ${ }^{(0)} A_{a}$ denotes an electromagnetic potential in $M_{\mathrm{o}}$. Again, we consider a monochromatic plane wave solution: ${ }^{(0)} A_{a}={ }^{(0)} \mathcal{A}_{a} e^{\mathrm{j}^{(0)} Q}$, where ${ }^{(0)} \mathcal{A}_{a}$ is a com- 
plex constant amplitude, and ${ }^{(0)} Q$ is a real phase scalar that satisfies ${ }^{(0)} n^{a} \partial_{a}{ }^{(0)} Q<0$ for an arbitrary observer with the 4 velocity ${ }^{(0)} n^{a}$ in the Minkowski spacetime and vanishes at the second covariant derivative. Also, the propagation vector for electromagnetic waves is defined as ${ }^{(0)} l^{a} \equiv \partial^{a(0)} Q$, which is a constant null vector. Note that one must distinguish the complex numbers $\mathrm{i}$ and $\mathrm{j}$ assigned to describe gravitational waves and electromagnetic waves, respectively: in particular, when they are mixed in quadratic forms, such as ij or ji. As i and $\mathrm{j}$ describe a phase shift by $\pi / 2$ with respect to the reference phase of each independent wave, they must be treated separately. We impose the commutativity of multiplication between $\mathrm{i}$ and $\mathrm{j}$; namely, $\mathrm{ij}=\mathrm{ji}$.

For an inertial observer with ${ }^{(0)} n^{a}$ living in the Minkowski spacetime, the propagation vector ${ }^{(0)} l^{a}$ can be decomposed into ${ }^{(0)} l^{a}={ }^{(0)} \omega_{\mathrm{e}}\left({ }^{(0)} n^{a}+{ }^{(0)} \lambda^{a}\right)$, where ${ }^{(0)} \omega_{\mathrm{e}}=$ ${ }^{(0)} n^{a} \partial_{a}{ }^{(0)} Q>0$ is the frequency of electromagnetic waves measured by the observer, and ${ }^{(0)} \lambda^{a}$ is a spatial unit vector orthogonal to ${ }^{(0)} n^{a}$ in the observer's point of view. Further, we impose an additional gauge condition ${ }^{(0)} A_{a}{ }^{(0)} n^{a}=0$. Then, electric and magnetic fields become ${ }^{(0)} E_{a}=\mathrm{j}^{(0)} \omega_{\mathrm{e}}{ }^{(0)} A_{a}$ and ${ }^{(0)} B_{a}=\mathrm{j}^{(0)} \omega_{\mathrm{e}}{ }^{(0)} \epsilon^{c}{ }_{a b}{ }^{(0)} \lambda^{b(0)} A_{c}$, respectively: it should be noted that ${ }^{(0)} A_{a}$ in our gauge conditions is proportional to a gauge-invariant quantity ${ }^{(0)} E_{a}$ or ${ }^{(0)} B_{a}$.

As shown in Appendix A, we can find a right-handed orthonormal frame $\left\{{ }^{(0)} n^{a},{ }^{(0)} p^{a},{ }^{(0)} s^{a},{ }^{(0)} \lambda^{a}\right\}$, in which the electric field is written in the form ${ }^{(0)} E_{a}=\left({ }^{(0)} \mathcal{E}_{(p)}{ }^{(0)} p_{a}\right.$ $\left.+\mathrm{j}^{(0)} \mathcal{E}_{(s)}{ }^{(0)} s_{a}\right) e^{\mathrm{j}^{(0)} Q}$, where ${ }^{(0)} \mathcal{E}_{(p)}$ and ${ }^{(0)} \mathcal{E}_{(s)}$ are real scalars, representing the axes of polarization ellipse. The ellipticity of polarization is defined as ${ }^{(0)} \chi \equiv \tan ^{-1}\left({ }^{(0)} \mathcal{E}_{(s)} /\right.$ $\left.{ }^{(0)} \mathcal{E}_{(p)}\right)$.

The intensity of electromagnetic waves on the plane orthogonal to a unit vector $z^{a}$ is obtained from the time average of a Poynting vector ${ }^{(0)} P^{a}={ }^{(0)} \epsilon^{a b c} \Re\left({ }^{(0)} E_{b}\right) \Re\left({ }^{(0)} B_{c}\right)$ contracted with $z^{a}$; i.e., Intensity $=\left\langle z^{a(0)} P_{a}\right\rangle$. The time average of a quantity $f(t)$ is defined as $\langle f(t)\rangle=\lim _{T \rightarrow \infty} \frac{1}{T} \int_{t}^{t+T}$ $f\left(t^{\prime}\right) d t^{\prime}$, where $t$ is the proper time measured by an observer with ${ }^{(0)} n^{a}[15]$.

Stokes parameters describe the polarization state of light that are obtained by measurements of intensities using optical devices, e.g., polarizers and waveplates: ${ }^{(0)} S_{0}$ is a measure of the total intensity of light, ${ }^{(0)} S_{1}$ and ${ }^{(0)} S_{2}$ jointly describe the linear polarization, and ${ }^{(0)} S_{3}$ describes the circular polarization. In the adapted frame $\left\{{ }^{(0)} n^{a},{ }^{(0)} p^{a},{ }^{(0)} s^{a},{ }^{(0)} \lambda^{a}\right\}$ for the electric field ${ }^{(0)} E_{a}=\left({ }^{(0)} \mathcal{E}_{(p)}{ }^{(0)} p_{a}+\mathrm{j}^{(0)} \mathcal{E}_{(s)}{ }^{(0)} s_{a}\right) e^{\mathrm{j}^{(0)} Q}$, we obtain ${ }^{(0)} S_{0}=\frac{1}{2}\left({ }^{(0)} \mathcal{E}_{(p)}^{2}+{ }^{(0)} \mathcal{E}_{(s)}^{2}\right),{ }^{(0)} S_{1}=\frac{1}{2}\left({ }^{(0)} \mathcal{E}_{(p)}^{2}\right.$ $\left.-{ }^{(0)} \mathcal{E}_{(s)}^{2}\right),{ }^{(0)} S_{2}=0$, and ${ }^{(0)} S_{3}={ }^{(0)} \mathcal{E}_{(p)}{ }^{(0)} \mathcal{E}_{(s)}$.

\section{Perturbation of electromagnetic waves}

Let us consider a perturbed spacetime $M_{\epsilon}$ in which the Riemann tensor does not vanish. Then Maxwell equations are written as:

$\nabla^{b} \nabla_{b} A_{a}=R_{a}^{b} A_{b}$

with the Lorenz gauge condition,

$\nabla^{a} A_{a}=0$

where $R_{a b}=R_{a c b}^{c}$ denotes the Ricci tensor. Eq. (1) presents inhomogeneous Maxwell equations, namely, electromagnetic wave equations extended to the curved (perturbed) spacetime $M_{\epsilon}$. Then we can write down a solution in the form:

$A_{a}=\mathcal{A}_{a} e^{\mathrm{j} Q}$,

where $\mathcal{A}_{a}$ and $Q$ correspond to the amplitude and phase of an electromagnetic wave, respectively in the Minkowski spacetime $M_{\mathrm{o}}$. Note that $\mathcal{A}_{a}$ is not constant in general, unlike its counterpart in $M_{\mathrm{o}}$, and that $l^{a} \equiv \nabla^{a} Q$ is not null in general, unlike its counterpart in $M_{\mathrm{o}}$. Then for the violation of the null condition, we define a quantity:

$v \equiv g_{a b} l^{a} l^{b}$

Also, $A_{a}$ is not a transverse wave due to $A_{a} l^{a} \neq 0$. Following from Eq. (3), the field strength tensor $F_{a b}=\nabla_{a} A_{b}-\nabla_{b} A_{a}$ is expressed in the same form:

$F_{a b}=\mathcal{F}_{a b} e^{\mathrm{j} Q}$,

where

$\mathcal{F}_{a b} \equiv 2 \nabla_{[a} \mathcal{A}_{b]}+2 \mathrm{j} l_{[a} \mathcal{A}_{b]}$

Let us consider a geodesic observer with $n^{a}$ in $M_{\epsilon}$, as mentioned in the previous section. We introduce the $3+1$ formalism to split a tensor into temporal and spatial parts, using a projection tensor $\gamma_{a b}$ defined as

$\gamma_{b}^{a} \equiv \delta_{b}^{a}+n^{a} n_{b}$

Then the propagation vector $l^{a}$ can be decomposed into the temporal component $\omega_{\mathrm{e}}$ and the spatial unit vector $\lambda^{a}$, given respectively by

$\omega_{\mathrm{e}} \equiv-n^{a} l_{a}$,
$\lambda^{a} \equiv \frac{\gamma^{a}{ }_{b} l^{b}}{\sqrt{\gamma_{c d} l^{c} l^{d}}}$. 
The electric and magnetic fields as measured by an observer with $n^{a}$ can be expressed in the same form as Eq. (3):

$$
\begin{aligned}
& E_{a}=\mathcal{E}_{a} e^{\mathrm{j} Q}, \\
& B_{a}=\mathcal{B}_{a} e^{\mathrm{j} Q},
\end{aligned}
$$

where

$$
\begin{aligned}
\mathcal{E}_{a} & =\mathcal{F}_{a b} n^{b}, \\
\mathcal{B}_{a} & =\frac{1}{2} \epsilon_{a}{ }^{b c} \mathcal{F}_{b c},
\end{aligned}
$$

where $\mathcal{F}_{a b}$ refers to Eq. (6), and $\epsilon_{a b c} \equiv n^{d} \varepsilon_{d a b c}$ denotes the 'spatial' Levi-Civita tensor while $\varepsilon_{d a b c}$ is the 'spacetime' Levi-Civita tensor.

According to Appendix A, we can introduce an adapted orthonormal frame $\left\{p^{a}, s^{a}\right\}$ into $M_{\epsilon}$ with no restriction, in which one can express

$\mathcal{E}^{a}=\mathcal{E}_{(p)} p^{a}+\mathrm{j} \mathcal{E}_{(s)} s^{a}$,

for the electric field Eq. (10), where the axes of polarization ellipse, $\mathcal{E}_{(p)}$ and $\mathcal{E}_{(s)}$ are real scalars given by

$$
\begin{aligned}
& \mathcal{E}_{(p)} \equiv \sqrt{\Re\left(\mathcal{E}_{a}\right) \Re\left(\mathcal{E}_{b}\right) g^{a b}}, \\
& \mathcal{E}_{(s)} \equiv \sqrt{\Im\left(\mathcal{E}_{a}\right) \Im\left(\mathcal{E}_{b}\right) g^{a b}},
\end{aligned}
$$

where $\mathfrak{R}()$ and $\mathfrak{\Im}()$ are defined by

$$
\begin{aligned}
& \Re(f)=\frac{1}{2}\left(f+f^{*}\right), \\
& \Im(f)=\frac{1}{2 \mathrm{j}}\left(f-f^{*}\right),
\end{aligned}
$$

where $f$ is a complex quantity and $*$ denotes the complex conjugate with respect to $\mathrm{j}$. There are multiple possible pairs of $\left\{p^{a}, s^{a}\right\}$ in $M_{\epsilon}$, but we choose one, whose values in the limit $\epsilon \rightarrow 0$ coincide with $\left\{p^{a}, s^{a}\right\}$ in $M_{\mathrm{o}}$. Also, we define the ellipticity as

$\chi \equiv \tan ^{-1}\left(\frac{\mathcal{E}_{(s)}}{\mathcal{E}_{(p)}}\right)$.

Note that the polarization plane spanned by $\left\{p^{a}, s^{a}\right\}$ is not orthogonal to $\lambda^{a}$ in general. Then for the violation of the transversity condition, we define a quantity:

$\tau \equiv \lambda^{a} E_{a}$

Now, let us consider a first-order perturbation of Maxwell equations. From Eq. (1) it becomes

$$
\partial^{b} \partial_{b}{ }^{(1)} A_{a}=2 C^{b c}{ }_{a} \partial_{c}{ }^{(0)} A_{b}+h^{b c} \partial_{b} \partial_{c}{ }^{(0)} A_{a},
$$

where

$$
\begin{aligned}
C^{a}{ }_{b c} & \equiv \mathcal{C}^{a}{ }_{b c} e^{\mathrm{i} P}, \\
\mathcal{C}^{a}{ }_{b c} & \equiv \frac{\mathrm{i}}{2}\left(\mathcal{H}^{a}{ }_{b} k_{c}+\mathcal{H}^{a}{ }_{c} k_{b}-\mathcal{H}_{b c} k^{a}\right) .
\end{aligned}
$$

Note that the perturbation of the Ricci tensor term from the right-hand side of Eq. (1) vanishes: it is due to the transversetraceless gauge. Assuming a solution in the form ${ }^{(1)} A_{a} \sim$ const. $\times e^{\mathrm{i} P} e^{\mathrm{j}(0)} Q$, it is obtained as:

$$
\begin{aligned}
{ }^{(1)} A_{a}= & \left(-\mathrm{i} \frac{\mathcal{C}^{b}{ }_{c a}{ }^{(0)} \mathcal{A}_{b}{ }^{(0)} l^{c}}{k^{d(0)} l_{d}}-\mathrm{ij} \frac{\mathcal{H}_{b c}{ }^{(0)} l^{b(0)} l^{c(0)} \mathcal{A}_{a}}{2 k^{d(0)} l_{d}}\right) \\
& \times e^{\mathrm{i} P e^{\mathrm{j}^{(0)} Q} .}
\end{aligned}
$$

Here we rule out the case of $k^{a(0)} l_{a}=0$, in which the righthand side of Eq. (21) vanishes and hence gravitational waves do not affect the electromagnetic wave to the first-order perturbation.

From Eq. (3) perturbation of $A_{a}$ to first order yields

$$
{ }^{(1)} A_{a}=\left({ }^{(1)} \mathcal{A}_{a}+\mathrm{j}^{(0)} \mathcal{A}_{a}{ }^{(1)} Q\right) e^{\mathrm{j}^{(0)} Q} .
$$

Matching this with Eq. (24), on the right-hand sides of the two equations, the first terms correspond to each other and so do the second terms: as $l^{a} \propto{ }^{(0)} \omega_{\mathrm{e}}$ (from Eq. (8)), the first terms are at $O\left({ }^{(0)} \omega_{\mathrm{e}}^{0}\right)$ while the second terms are at $O\left({ }^{(0)} \omega_{\mathrm{e}}^{1}\right)$, where ${ }^{(0)} \omega_{\mathrm{e}}$ serves as an order-counting parameter in the geometrical-optics approach [16]. Then the perturbations of amplitude and phase, ${ }^{(1)} \mathcal{A}$ and ${ }^{(1)} Q$ can be identified respectively as:

$$
\begin{aligned}
{ }^{(1)} Q & =-\mathrm{i} \frac{\mathcal{H}_{b c}{ }^{(0)} l^{b(0)} l^{c}}{2 k^{d(0)} l_{d}} e^{\mathrm{i} P}, \\
{ }^{(1)} \mathcal{A}_{a} & =-\mathrm{i} \frac{\mathcal{C}^{b}{ }^{b}{ }^{(0)} \mathcal{A}_{b}{ }^{(0)} l^{c}}{k^{d(0)} l_{d}} e^{\mathrm{i} P} .
\end{aligned}
$$

Here one should be careful about the denominators, expressed by $\sim k^{a(0)} l_{a}=\omega_{\mathrm{g}}{ }^{(0)} \omega_{\mathrm{e}}(-1+\cos \theta)$, where $\theta$ is the angle between the propagation directions of gravitational and electromagnetic waves. As $\theta \rightarrow 0$, the first term inside the round brackets of Eq. (24) diverges while the second term converges; hence, separation of the terms by order of ${ }^{(0)} \omega_{\mathrm{e}}$ fails. Then the value of $\theta$ for the geometrical-optics approach to be valid is given by

$\theta \gtrsim \sqrt{2 \frac{\omega_{\mathrm{g}}}{{ }^{(0)} \omega_{\mathrm{e}}}}$.

Substituting Eq. (26) into the first-order perturbation of $v$ from Eq. (4) through $l^{a}=\nabla^{a} Q$, we obtain

${ }^{(1)} v=0$. 
This means that the null condition for the electromagnetic wave is not violated to the first-order perturbation. In the same manner, from perturbation of Eq. (8), we obtain the fractional perturbation of the electromagnetic wave frequency, given by

$\frac{{ }^{(1)} \omega_{\mathrm{e}}}{{ }^{(0)} \omega_{\mathrm{e}}}=-\frac{1}{2 \Theta} \mathcal{H}_{a b}{ }^{(0)} \lambda^{a(0)} \lambda^{b} e^{\mathrm{i} P}$,

where $\Theta \equiv 1-\kappa^{a(0)} \lambda_{a}=1-\cos \theta$. Note that ${ }^{(1)} \nu$ and ${ }^{(1)} \omega_{\mathrm{e}}$ are gauge-invariant as they are constant scalars evaluated in $M_{\mathrm{O}}$.

From Eq. (6) perturbation of $\mathcal{F}_{a b}$ to first order yields

${ }^{(1)} \mathcal{F}_{a b}=2 \partial_{[a}{ }^{(1)} \mathcal{A}_{b]}+2 \mathrm{j}\left(\partial_{[a}{ }^{(1)} Q\right){ }^{(0)} \mathcal{A}_{b]}+2 \mathrm{j}^{(0)} l_{[a}{ }^{(1)} \mathcal{A}_{b]}$.

Here the first term on the right-hand side, being at $O\left(\omega_{\mathrm{g}}{ }^{(0)} \omega_{\mathrm{e}}^{0}\right)$, can be ignored in comparison with the other terms at $O\left({ }^{(0)} \omega_{\mathrm{e}}^{1}\right)$ in the geometrical-optics approximation, provided that ${ }^{(0)} \omega_{\mathrm{e}}^{1} \gg \omega_{\mathrm{g}}{ }^{(0)} \omega_{\mathrm{e}}^{0}$. Now, from Eqs. (12) and (13) the firstorder perturbations of $\mathcal{E}_{a}$ and $\mathcal{B}_{a}$ are given by

${ }^{(1)} \mathcal{E} a={ }^{(1)} \mathcal{F}_{a b}{ }^{(0)} n^{b}$,

${ }^{(1)} \mathcal{B} a=\frac{1}{2}{ }^{(0)} \epsilon^{b c}{ }_{a}{ }^{(1)} \mathcal{F}_{b c}-{ }^{(0)} \epsilon^{b c}{ }_{a} h^{d}{ }_{b}{ }^{(0)} \mathcal{F}_{d c}$.

In Eq. (33) we have used the fact that the perturbation of the spatial Levi-Civita tensor vanishes:

${ }^{(1)} \epsilon_{a b c}=0$,

which is shown in Appendix C.

Using these together with Eqs. (23), (26), (27), (31) and (32) for the perturbations of $\mathcal{E}_{(p)}$ and $\mathcal{E}_{(s)}$ from Eqs. (15) and (16), we obtain the fractional perturbations of the axes of polarization ellipse, given by

$\frac{{ }^{(1)} \mathcal{E}_{(p)}}{{ }^{(0)} \mathcal{E}_{(p)}}=\frac{{ }^{(1)} \mathcal{E}_{(s)}}{{ }^{(0)} \mathcal{E}_{(s)}}=-\frac{1}{2 \Theta} \mathcal{H}_{a b}{ }^{(0)} \lambda^{a(0)} \lambda^{b} e^{\mathrm{i} P}$

Due to this, however, the perturbation of the ellipticity $\chi$ from Eq. (19) vanishes:

${ }^{(1)} \chi=0$.

This means that the ellipticity is maintained while the perturbed axes of polarization ellipse oscillate with gravitational waves: they expand and shrink periodically together.

Using Eqs. (9), (10), (12) and (32) for Eq. (20), we obtain the perturbation of $\tau$ :

${ }^{(1)} \tau=0$.
This means that the transversity condition for the electromagnetic wave is not violated to the first-order perturbation. Note that ${ }^{(1)} \mathcal{E}_{(p)},{ }^{(1)} \mathcal{E}_{(s)},{ }^{(1)} \chi$, and ${ }^{(1)} \tau$ are gauge-invariant as they are constant scalars evaluated in $M_{\mathrm{O}}$.

\section{Application: observation of gravitational waves}

With regard to the observation of gravitational waves, one can consider the measurement of Stokes parameters and their perturbations. This requires that the Stokes parameters be measured in a perturbed spacetime $M_{\epsilon}$. For this purpose, we introduce another right-handed orthonormal frame $\left\{n^{a}, x^{a}, y^{a}, z^{a}\right\}$, where $z^{a}$ is directed along the time-averaged Poynting vector $P^{a}$ for electromagnetic waves, given by

$z^{a} \equiv \frac{\left\langle P^{a}\right\rangle}{\sqrt{g_{b c}\left\langle P^{b}\right\rangle\left\langle P^{c}\right\rangle}}$.

Here the Poynting vector $P^{a}$ is defined as the cross product of the real electric and magnetic fields in $M_{\epsilon}$ :

$P^{a}=\epsilon^{a b c} \Re\left(E_{b}\right) \Re\left(B_{c}\right)$.

And the time average of a quantity $f(t)$ is defined as

$\langle f(t)\rangle=\frac{1}{T} \int_{t}^{t+T} f\left(t^{\prime}\right) d t^{\prime}$

where $t$ is the proper time measured by an observer with $n^{a}$, and $T$ is a time scale such that $\omega_{\mathrm{e}} T \gg 1 \gg \omega_{\mathrm{g}} T$ with $T$ covering a finite number of oscillation periods of the electromagnetic fields; therefore, defined through the time average, $z^{a}$ contains the perturbation oscillating at $\omega_{\mathrm{g}}$, exhibiting the effects of gravitational waves, while its unperturbed part is constant. $x^{a}$ and $y^{a}$ are perturbed accordingly while being orthogonal to $z^{a}$ and to each other. We consider that the frame $\left\{n^{a}, x^{a}, y^{a}, z^{a}\right\}$ defined in this manner is experimentally feasible as it is determined naturally based on an observable; namely, the time-averaged Poynting vector.

In this frame, the Stokes parameters are expressed as:

$4 \pi S_{I}=\frac{1}{2}\left\langle\Re\left\{\mathfrak{p}_{I}^{a b}\left(\mathcal{E}_{a} \mathcal{B}_{b}^{*}+\mathcal{E}_{a} \mathcal{B}_{b} e^{2 \mathrm{j} Q}\right)\right\}\right\rangle$,

where $I=0,1,2,3$, and $\mathfrak{p}_{I}^{a b}$ denote projections defined by

$\mathfrak{p}_{0}^{a b} \equiv x^{a} y^{b}-y^{a} x^{b}$,

$\mathfrak{p}_{1}^{a b} \equiv x^{a} y^{b}+y^{a} x^{b}$,

$\mathfrak{p}_{2}^{a b} \equiv-x^{a} x^{b}+y^{a} y^{b}$,

$\mathfrak{p}_{3}^{a b} \equiv-\mathrm{j}\left(x^{a} x^{b}+y^{a} y^{b}\right)$. 
It should be noted that the Stokes parameters $S_{I}$ in $M_{\epsilon}$, being defined through the time average as above, contain the perturbations oscillating at $\omega_{\mathrm{g}}$, induced by gravitational waves, unlike their counterparts in $M_{\mathrm{o}}$. Using rotational properties of $S_{1}$ and $S_{2}$, we set $\left\{x^{a}, y^{a}\right\}$ such that Stokes parameter $S_{2}$ vanishes. There are two possible sets of $\left\{n^{a}, x^{a}, y^{a}, z^{a}\right\}$ in $M_{\epsilon}$ satisfying this, but we choose one, whose values in the limit $\epsilon \rightarrow 0$ coincide with $\left\{{ }^{(0)} n^{a},{ }^{(0)} p^{a},{ }^{(0)} s^{a},{ }^{(0)} \lambda^{a}\right\}$ in $M_{\mathrm{o}}$.

Now, we consider perturbations of the Stokes parameters. For this sake, we need a perturbation of the spatial LeviCivita tensor, as given by Eq. (34), together with the components along ${ }^{(0)} p^{a}$ and ${ }^{(0)} s^{a}$ of the perturbations of the frame $\left\{x^{a}, y^{a}\right\}$,

$$
\begin{aligned}
{ }^{(1)} x^{a(0)} p_{a} & =-\frac{1}{2} \mathcal{H}_{a b}{ }^{(0)} p^{a(0)} p^{b} e^{\mathrm{i} P}, \\
{ }^{(1)} y^{a(0)} s_{a} & =-\frac{1}{2} \mathcal{H}_{a b}{ }^{(0)} s^{a(0)} s^{b} e^{\mathrm{i} P},
\end{aligned}
$$

the derivations of which are given in Appendix D. Using these, we obtain the fractional perturbations of the Stokes parameters from Eq. (41), given by

$$
\begin{aligned}
& \frac{{ }^{(1)} S_{0}}{{ }^{(0)} S_{0}}=-\frac{1}{\Theta} \mathcal{H}_{a b}{ }^{(0)} \lambda^{a(0)} \lambda^{b} e^{\mathrm{i} P}, \\
& \frac{{ }^{(1)} S_{1}}{{ }^{(0)} S_{1}}=-\frac{1}{\Theta} \mathcal{H}_{a b}{ }^{(0)} \lambda^{a(0)} \lambda^{b} e^{\mathrm{i} P}, \\
& { }^{(1)} S_{2}=0, \\
& \frac{{ }^{(1)} S_{3}}{{ }^{(0)} S_{3}}=-\frac{1}{\Theta} \mathcal{H}_{a b}{ }^{(0)} \lambda^{a(0)} \lambda^{b} e^{\mathrm{i} P} .
\end{aligned}
$$

Note that these are gauge-invariant quantities as they are constant scalars evaluated in $M_{0}$.

In relation to the observation of gravitational waves, let us discuss the antenna patterns for the measurement of $h$ through the Stokes parameters, defined by

$h \equiv-\frac{1}{\Theta} \mathcal{H}_{a b}{ }^{(0)} \lambda^{a(0)} \lambda^{b} e^{\mathrm{i} P}$.

Now, we introduce an adapted frame for gravitational waves. As shown in Appendix B, one can find a right-handed orthonormal frame $\left\{{ }^{(0)} n^{a}, u^{a}, v^{a}, \kappa^{a}\right\}$, in which the amplitude of gravitational waves is expressed as:

$\mathcal{H}_{a b}=\mathcal{H}_{+} e_{a b}^{+}+\mathrm{i} \mathcal{H}_{\times} e_{a b}^{\times}$,

where $\mathcal{H}_{+}$and $\mathcal{H}_{\times}$are real scalars, the projections of the amplitude onto the polarization tensors $e_{a b}^{+}$and $e_{a b}^{\times}$, respectively, defined by

$e_{a b}^{+} \equiv u_{a} u_{b}-v_{a} v_{b}$,

$e_{a b}^{\times} \equiv u_{a} v_{b}+v_{a} u_{b}$.
Then the Euler angles $(\phi, \theta, \psi)$ as defined in Refs. $[17,18]$ yield the following relations between the adapted spatial frames $\left\{{ }^{(0)} p^{a},{ }^{(0)} s^{a},{ }^{(0)} \lambda^{a}\right\}$ and $\left\{u^{a}, v^{a}, \kappa^{a}\right\}$ for electromagnetic and gravitational waves, respectively:

$u^{a}=R\left({ }^{(0)} \lambda^{a}, \phi\right) R\left({ }^{(0)} s^{a}, \theta\right) R\left({ }^{(0)} \lambda^{a}, \psi\right){ }^{(0)} p^{a}$,

$v^{a}=R\left({ }^{(0)} \lambda^{a}, \phi\right) R\left({ }^{(0)} s^{a}, \theta\right) R\left({ }^{(0)} \lambda^{a}, \psi\right){ }^{(0)} s^{a}$,

$\kappa^{a}=R\left({ }^{(0)} \lambda^{a}, \phi\right) R\left({ }^{(0)} s^{a}, \theta\right) R\left({ }^{(0)} \lambda^{a}, \psi\right){ }^{(0)} \lambda^{a}$,

where $R\left(\beta^{a}, \alpha\right)$ denotes a rotation of a vector by the angle $\alpha$ with respect to the axis $\beta^{a}$.

Substituting Eq. (53) into Eq. (52), we obtain

$h=\left(\mathcal{H}_{+} F_{+}+\mathrm{i} \mathcal{H}_{\times} F_{\times}\right) e^{i P}$,

where $F_{+}$and $F_{\times}$are the antenna patterns for + and $\times$polarization states, respectively, given by

$F_{+}=-2 \cos ^{2}(\theta / 2) \cos (2 \psi)$,

$F_{\times}=2 \cos ^{2}(\theta / 2) \sin (2 \psi)$.

These are in agreement with Refs. [19-22] , although our angles $\theta$ and $\psi$ are defined in a slightly different manner than the ones in the literature. In Fig. 1 are presented the antenna patterns of $\left|F_{+}\right|$and $\left|F_{\times}\right|$as viewed in consideration with gravitational waves, which propagate along the direction of the $\boldsymbol{\kappa}$-axis while being polarized in the $\boldsymbol{u} \boldsymbol{v}$-plane in a quadrupole manner. We evaluate the sky averages of the following quantities over $(\phi, \theta, \psi)$ :

$\left\langle F_{+} F_{+}\right\rangle=\frac{2}{3}$,

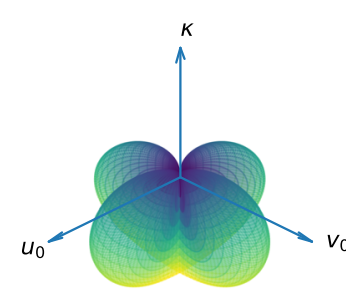

$\left|F_{+}\right|$

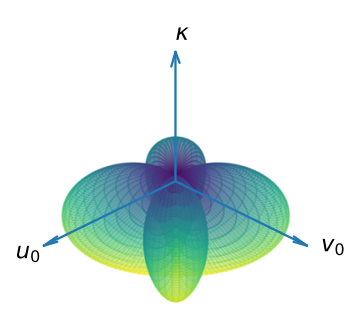

$\left|F_{\times}\right|$

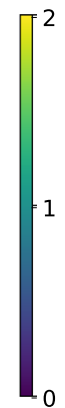

Fig. 1 Antenna patterns of $\left|F_{+}\right|$and $\left|F_{\times}\right|$for the measurement of $h$; as viewed in consideration with gravitational waves propagating along the direction of the $\boldsymbol{\kappa}$-axis while being polarized in the $\boldsymbol{u} \boldsymbol{v}$-plane in a quadrupole manner. The frame $\left\{\boldsymbol{u}_{0}, \boldsymbol{v}_{0}, \boldsymbol{\kappa}\right\}$ is defined from an adapted frame $\{\boldsymbol{u}, \boldsymbol{v}, \boldsymbol{\kappa}\}$ with $\psi=0$. In this frame, the polar angle is defined as $\vartheta=\pi-\theta$, where $\theta$ refers to the angle between the propagation directions of gravitational and electromagnetic waves, whereas the azimuthal angle is defined as $\varphi=\pi / 2+\psi$ 
$\left\langle F_{\times} F_{\times}\right\rangle=\frac{2}{3}$

$\left\langle F_{+} F_{\times}\right\rangle=0$.

Then the angular efficiency factor as defined in Ref. [23] is

$F \equiv\left\langle F_{+} F_{+}\right\rangle+\left\langle F_{\times} F_{\times}\right\rangle=\frac{4}{3}$.

And the sky average of $|h|^{2}$ is given by

$\left\langle|h|^{2}\right\rangle=\frac{2}{3}\left(\mathcal{H}_{+}^{2}+\mathcal{H}_{\times}^{2}\right)$.

\section{Discussion}

We have worked out a perturbation of electromagnetic waves due to gravitational waves from the perturbed Maxwell equations, as given by Eqs. (25)-(27). The perturbation has two oscillatory parts from electromagnetic and gravitational waves. Inspecting it closely, the amplitude of the perturbed electromagnetic waves in general contains the perturbations of amplitude and phase mixed together in it. Then to disentangle the mixed perturbations from each other, we invoke the geometrical-optics approach, adopting the frequency of electromagnetic waves as an order-counting parameter. However, it should be noted that the geometrical-optics approach can break down if the propagation directions of electromagnetic and gravitational waves are extremely close to each other; so close as to almost coincide, as shown by our analysis with Eq. (28) .

From this perturbation analysis, we have found that the null condition for the electromagnetic wave is not violated to the first-order perturbation, as shown by Eq. (29). Also, we have obtained the fractional perturbation of the electromagnetic wave frequency, as given by Eq. (30), and confirmed that it is equivalent to the gravitational-wave-induced redshift in the literature [5]. In addition, the axes of polarization ellipse defined via the perturbed electric field exhibit the oscillatory feature of gravitational waves, as shown by Eq. (35): they expand and shrink periodically together. However, the ellipticity and the orthogonality between the polarization ellipse and the propagation direction of the electromagnetic wave are preserved to the first-order perturbation due to gravitational waves, as evidenced by Eqs. (36 and (37).

We have employed Stokes parameters as optical observables containing the gravitational wave signals. The measurement of the Stokes parameters requires a suitable orthonormal frame in which the parameters are expressed. To this end, we set one spatial unit vector to be along the propagation direction of the time-averaged Poynting vector, and arrange the other two vectors perpendicular to this such that the Stokes parameter $S_{2}$ vanishes. We have obtained the fractional perturbations of the other non-vanishing Stokes parameters, as given by Eqs. (48), (49) and (51), which have turned out to be all identical. Interestingly, apart from the factor 2 , these are also identical to the fractional perturbations of the electromagnetic wave frequency and the axes of polarization ellipse, as given by Eqs. (30) and (35). The antenna patterns defined through the Stokes parameters, as given by Eqs. (60 and (61), show that the detectability of gravitational wave signals vanishes when the propagation directions of gravitational and electromagnetic waves are opposite to each other, whereas the detectability becomes the maximum when the two waves propagate in the same direction, as described in Fig. 1.

Our analysis might find its application to some practical issue in relation to Cosmic Microwave Background (CMB) anisotropies, characterized by scalar perturbations due to temperature fluctuations and tensor perturbations due to generation of polarization. As for the latter, free charges, being agitated by primordial gravitational waves propagating through the CMB plasma, rescatter electromagnetic radiation, and this imprints a characteristic pattern of linear polarization on the CMB map, represented by E-modes and Bmodes, which are derived by means of the Stokes parameters [24-30]. However, if other gravitational waves from different sources pass through while observing the CMB radiation, the Stokes parameters will be perturbed as given by Eqs. (48), (49) and (51), causing the polarization pattern to change through the perturbed E-modes and B-modes accordingly. Therefore, for accurate measurement of the effects from primordial gravitational waves alone, the effects from other gravitational waves should be identified and disentangled carefully, based on our analysis of polarization.

In this work, we have investigated the effect of gravitational waves on one light ray. But it will also be fairly interesting to study the effect of gravitational waves in a situation where two or more light rays interfere with each other. With respect to this, most analyses regarding the interferometry in the literature have concentrated on the phase perturbation so far. However, a more complete analysis of the interferometry would require consideration of the effect from the amplitude perturbation as well. In addition, it will be interesting to study the effect of gravitational waves in a situation where the polarization directions of two or more light rays are not aligned. We leave discussion of all these for the future research.

Acknowledgements C. Park appreciates Gungwon Kang for valuable discussions. C. Park was supported in part by the Basic Science Research Program through the National Research Foundation of Korea (NRF) funded by the Ministry of Education (NRF2018R1D1A1B07041004), and by the National Institute for Mathematical Sciences (NIMS) funded by Ministry of Science and ICT (B20710000). D.-H. Kim was supported by the Basic Science Research 
Program through the National Research Foundation of Korea (NRF) funded by the Ministry of Education (NRF-2018R1D1A1B07051276).

Data Availability Statement This manuscript has no associated data or the data will not be deposited. [Authors' comment: This work presents completely analytical results with no data associated.]

Open Access This article is licensed under a Creative Commons Attribution 4.0 International License, which permits use, sharing, adaptation, distribution and reproduction in any medium or format, as long as you give appropriate credit to the original author(s) and the source, provide a link to the Creative Commons licence, and indicate if changes were made. The images or other third party material in this article are included in the article's Creative Commons licence, unless indicated otherwise in a credit line to the material. If material is not included in the article's Creative Commons licence and your intended use is not permitted by statutory regulation or exceeds the permitted use, you will need to obtain permission directly from the copyright holder. To view a copy of this licence, visit http://creativecomm ons.org/licenses/by/4.0/.

Funded by SCOAP ${ }^{3}$.

\section{Appendix A: Adapted frame for electric field}

Given an electric field $E_{a}=\mathcal{E}_{a} e^{\mathrm{j} Q}$, one can consider that there always exists a suitable phase $\alpha$ to make

$E_{a}=\mathcal{E}_{a} e^{-\mathrm{j} \alpha} e^{\mathrm{j}(Q+\alpha)}=\mathcal{E}_{a}^{\prime} e^{\mathrm{j} Q^{\prime}}$,

such that

$\Re_{\mathrm{j}}\left(\mathcal{E}_{a}^{\prime}\right) \Im_{\mathrm{j}}\left(\mathcal{E}_{b}^{\prime}\right) g^{a b}=0$,

where $\Re_{j}()$ and $\Im_{j}()$ are defined by

$\Re_{\mathrm{j}}(f)=\frac{1}{2}\left(f+f^{*}\right)$,

$\Im_{\mathrm{j}}(f)=\frac{1}{2 \mathrm{j}}\left(f-f^{*}\right)$

where $f$ is a complex quantity and $*$ denotes the complex conjugate with respect to $\mathrm{j}$. Dropping the sign ' from the lefthand side, Eq. (A2) is the orthogonality condition for the decomposed parts of $\mathcal{E}^{a}$; namely, $\Re_{\mathrm{j}}\left(\mathcal{E}^{a}\right) \perp \Im_{\mathrm{j}}\left(\mathcal{E}^{a}\right)$. Out of this, we can construct an orthonormal frame $\left\{p^{a}, s^{a}\right\}$ by

$p^{a} \equiv \frac{\Re_{\mathrm{j}}\left(\mathcal{E}^{a}\right)}{\left\|\Re_{\mathrm{j}}(\mathcal{E})\right\|}$,

$s^{a} \equiv \frac{\Im_{\mathrm{j}}\left(\mathcal{E}^{a}\right)}{\left\|\Im_{\mathrm{j}}(\mathcal{E})\right\|}$,

where $\|X\| \equiv \sqrt{X^{a} X^{b} g_{a b}}$ for a vector $X^{a}$. In this frame, $\mathcal{E}^{a}$ is expressed in the form:

$\mathcal{E}^{a}=\mathcal{E}_{(p)} p^{a}+\mathrm{j} \mathcal{E}_{(s)} s^{a}$,

where $\mathcal{E}_{(p)} \equiv\left\|\Re_{\mathrm{j}}(\mathcal{E})\right\|$ and $\mathcal{E}_{(s)} \equiv\left\|\Im_{\mathrm{j}}(\mathcal{E})\right\|$ are real scalars.

\section{Appendix B: Adapted frame for gravitational waves}

Given a gravitational wave $h_{a b}=\mathcal{H}_{a b} e^{\mathrm{i} P}$, one can consider that there always exists a suitable phase $\beta$ to make

$h_{a b}=\mathcal{H}_{a b} e^{-\mathrm{i} \beta} e^{\mathrm{i}(P+\beta)}=\mathcal{H}_{a b}^{\prime} e^{\mathrm{i} P^{\prime}}$,

such that

$\Re_{\mathrm{i}}\left(\mathcal{H}_{a b}^{\prime}\right) \Im_{\mathrm{i}}\left(\mathcal{H}_{c d}^{\prime}\right) g^{a c} g^{b d}=0$

where $\Re_{\mathrm{i}}()$ and $\Im_{\mathrm{i}}()$ are defined by

$\Re_{\mathrm{i}}(f)=\frac{1}{2}\left(f+f^{*}\right)$,

$\Im_{\mathrm{i}}(f)=\frac{1}{2 \mathrm{i}}\left(f-f^{*}\right)$,

where $f$ is a complex quantity and $*$ denotes the complex conjugate with respect to i. Dropping the sign ' from the left-hand side, Eq. (B2) is the orthogonality condition for the decomposed parts of $\mathcal{H}_{a b}$; namely, $\Re_{\mathrm{i}}\left(\mathcal{H}_{a b}\right) \perp \Im_{\mathrm{i}}\left(\mathcal{H}_{a b}\right)$. Out of this, we can construct a tensor basis $\left\{e_{a b}^{+}, e_{a b}^{\times}\right\}$by

$e_{a b}^{+}=\sqrt{2} \frac{\Re_{\mathrm{i}}\left(\mathcal{H}_{a b}\right)}{\left\|\Re_{\mathrm{i}}(\mathcal{H})\right\|}$,

$e_{a b}^{\times}=\sqrt{2} \frac{\Im_{\mathrm{i}}\left(\mathcal{H}_{a b}\right)}{\left\|\Im_{\mathrm{i}}(\mathcal{H})\right\|}$,

where $\|T\| \equiv \sqrt{T_{a b} T_{c d} g^{a c} g^{b d}}$ for a rank $(0,2)$ tensor $T_{a b}$. In this basis, $\mathcal{H}_{a b}$ is expressed in the form:

$\mathcal{H}_{a b}=\mathcal{H}_{+} e_{a b}^{+}+\mathrm{i} \mathcal{H}_{\times} e_{a b}^{\times}$,

where $\mathcal{H}_{+} \equiv\left\|\Re_{\mathrm{i}}(\mathcal{H})\right\| / \sqrt{2}$ and $\mathcal{H}_{\times} \equiv\left\|\Im_{\mathrm{i}}(\mathcal{H})\right\| / \sqrt{2}$ are real scalars.

We can create an orthonormal frame $\left\{u^{a}, v^{a}\right\}$ such that linear combinations of $u^{a}$ and $v^{a}$ satisfy the following equalities in view of Eqs. (B5)-(B7):

$\Re_{\mathrm{i}}\left(\mathcal{H}_{a b}\right)\left(c_{1} u^{a}+c_{2} \mathrm{i} v^{a}\right)=c_{3} u_{b}+c_{4} \mathrm{i} v_{b}$,

$\Im_{\mathrm{i}}\left(\mathcal{H}_{a b}\right)\left(c_{1} u^{a}+c_{2} \mathrm{i} v^{a}\right)=c_{5} u_{b}+c_{6} \mathrm{i} v_{b}$,

where $c_{1}, c_{2}, c_{3}, c_{4}, c_{5}, c_{6}$ are undetermined coefficients. Contracting both sides of Eqs. (B8) and (B9) with $c_{1} u^{b}+$ $c_{2} \mathrm{i} v^{b}$, and using $u^{b} u_{b}=v^{b} v_{b}=1$ and $u^{b} v_{b}=0$, we find

$\Re_{\mathrm{i}}\left(\mathcal{H}_{a b}\right)\left[\left(u^{a} u^{b}-v^{a} v^{b}\right)+\mathrm{i}\left(u^{a} v^{a}+v^{a} u^{a}\right)\right]=c_{3}-c_{4}$,

$\Im_{\mathrm{i}}\left(\mathcal{H}_{a b}\right)\left[\left(u^{a} u^{b}-v^{a} v^{b}\right)+\mathrm{i}\left(u^{a} v^{a}+v^{a} u^{a}\right)\right]=c_{5}-c_{6}$, 
where setting $c_{1}=c_{2}$ has simplified the equalities. Now, defining the tensor basis by

$e_{a b}^{+} \equiv u_{a} u_{b}-v_{a} v_{b}$,

$e_{a b}^{\times} \equiv u_{a} v_{b}+v_{a} u_{b}$,

and using Eqs. (B5)-(B7) for Eqs. (B10) and (B11), we determine $c_{1}=c_{2}=1, c_{3}=-c_{4}=\mathcal{H}_{+}, c_{5}=-c_{6}=\mathrm{i} \mathcal{H}_{\times}$. Then from Eqs. (B10) and (B11) we establish

$\mathcal{H}_{a b}\left(e^{+a b}+\mathrm{i} e^{\times a b}\right)=2\left(\mathcal{H}_{+}+\mathrm{i} \mathcal{H}_{\times}\right)$,

which is equivalent to Eq. (B7).

\section{Appendix C: Perturbation of Levi-Civita tensor}

The spacetime Levi-Civita tensor $\varepsilon_{a b c d}$ is normalized by

$-4 !=\varepsilon_{a b c d} \varepsilon_{e f g h} g^{a e} g^{b f} g^{c g} g^{d h}$.

From this perturbation of the tensor to first order yields

${ }^{(1)} \varepsilon_{a b c d}=\frac{1}{2} h^{e} e^{(0)} \varepsilon_{a b c d}$.

By our choice of the perturbation gauge, $h^{a}{ }_{a}=0,{ }^{(1)} \varepsilon_{a b c d}$ vanishes. Then this leads to the perturbation of the spatial Levi-Civita tensor $\epsilon_{a b c} \equiv n^{d} \varepsilon_{d a b c}$ (for a geodesic observer with the 4-velocity $n^{a}$ ) vanishing too:

${ }^{(1)} \epsilon_{a b c}={ }^{(0)} n^{d(1)} \varepsilon_{d a b c}+{ }^{(0)} \varepsilon_{d a b c}{ }^{(1)} n^{d}=0$.

\section{Appendix D: Perturbation of normalized frame}

The orthonormal frame $\left\{x^{a}, y^{a}\right\}$ for the observation of Stokes parameters must satisfy

$x^{a} x^{b} g_{a b}=y^{a} y^{b} g_{a b}=1$,

$x^{a} y^{b} g_{a b}=0$.

With no perturbation, the frame $\left\{x^{a}, y^{a}\right\}$ would tend to $\left\{{ }^{(0)} p^{a},{ }^{(0)} s^{a}\right\}$ in the background. Then from the normalization condition (D1), perturbation of $x^{a}$ and $y^{a}$ to first order yields

$$
\begin{aligned}
& { }^{(1)} x^{a(0)} p_{a}=-\frac{1}{2} h_{a b}{ }^{(0)} p^{a(0)} p^{b}, \\
& { }^{(1)} y^{a(0)} s_{a}=-\frac{1}{2} h_{a b}{ }^{(0)} s^{a(0)} s^{b} .
\end{aligned}
$$

\section{References}

1. M. Rakhmanov, Response of LIGO to gravitational waves at high frequencies and in the vicinity of the FSR $(37.5 \mathrm{kHz})$. Technical notes LIGO-T060237-x0 (2007)

2. M. Rakhmanov, J.D. Romano, J.T. Whelan, High-frequency corrections to the detector response and their effect on searches for gravitational waves. Class. Quantum Gravity 25(18), 184017 (2008)

3. M. Rakhmanov, On the round-trip time for a photon propagating in the field of a plane gravitational wave. Class. Quantum Gravity 26(15), 155010 (2009)

4. K.S. Thorne, R.D. Blandford, Modern Classical Physics: Optics, Fluids, Plasmas, Elasticity, Relativity, and Statistical Physics (Princeton University Press, Princeton, 2017)

5. S. Detweiler, Pulsar timing measurements and the search for gravitational waves. Astrophys. J. 234, 1100 (1979)

6. R.W. Hellings, G.S. Downs, Upper limits on the isotropic gravitational radiation background from pulsar timing analysis. Astrophys. J. 265, L39 (1983)

7. E. Montanari, On the propagation of electromagnetic radiation in the field of a plane gravitational wave. Class. Quantum Gravity 15(8), 2493-2507 (1998)

8. M. Calura, E. Montanari, Exact solution to the homogeneous Maxwell equations in the field of a gravitational wave in linearized theory. Class. Quantum Gravity 16(2), 643-652 (1999)

9. M. Halilsoy, O. Gurtug, Search for gravitational waves through the electromagnetic Faraday rotation. Phys. Rev. D 75(12), 124021 (2007)

10. S. Hacyan, Electromagnetic waves and Stokes parameters in the wake of a gravitational wave. Gen. Relativ. Gravit. 44(11), 2923$2931(2012)$

11. S. Hacyan, Effects of gravitational waves on the polarization of pulsars. Int. J. Mod. Phys. A 31(02n03), 1641023 (2016)

12. F. Cabral, F.S.N. Lobo, Gravitational waves and electrodynamics: new perspectives. Eur. Phys. J. C 77(4), 237 (2017)

13. J.M. Stewart, M. Walker, R. Penrose, Perturbations of space-times in general relativity. Proc. R. Soc. Lond. A Math. Phys. Sci. 341(1624), 49-74 (1974)

14. J.M. Stewart, Perturbations of Friedmann-Robertson-Walker cosmological models. Class. Quantum Gravity 7(7), 1169-1180 (1990)

15. J.R. Reitz, F.J. Milford, R.W. Christy, Foundations of Electromagnetic Theory (Pearson/Addison-Wesley, San Francisco, 2009). OCLC:252799997

16. S.R. Dolan, Geometrical optics for scalar, electromagnetic and gravitational waves on curved spacetime. Int. J. Mod. Phys. D 27(11), 1843010 (2018)

17. H. Goldstein, C.P. Poole, J.L. Safko. Classical Mechanics, 3 edn (Addison Wesley, San Francisco, 2002). OCLC:248389949

18. Archana Pai, Sanjeev Dhurandhar, Sukanta Bose, Data-analysis strategy for detecting gravitational-wave signals from inspiraling compact binaries with a network of laser-interferometric detectors. Phys. Rev. D 64(4), 042004 (2001)

19. Sydney J. Chamberlin, Xavier Siemens, Stochastic backgrounds in alternative theories of gravity: overlap reduction functions for pulsar timing arrays. Phys. Rev. D 85(8), 082001 (2012)

20. M.E. da Silva Alves, M. Tinto, Pulsar timing sensitivities to gravitational waves from relativistic metric theories of gravity. Phys. Rev. D 83(12), 123529 (2011)

21. K.J. Lee, F.A. Jenet, R.H. Price, Pulsar timing as a probe of non-Einsteinian polarizations of gravitational waves. Astrophys. J. 685(2), 1304-1319 (2008) 
22. N. Yunes, X. Siemens, Gravitational-wave tests of general relativity with ground-based detectors and pulsar-timing arrays. Living Rev. Relativ. 16(1), 9 (2013)

23. M. Maggiore, Gravitational Waves: Volume 1: Theory and Experiments, vol. 1 (Oxford University Press, Oxford, 2007)

24. J.M. Kovac, E.M. Leitch, C. Pryke, J.E. Carlstrom, N.W. Halverson, W.L. Holzapfel, Detection of polarization in the cosmic microwave background using DASI. Nature 420(6917), 772-787 (2002)

25. H. Wayne, M. White, CMB anisotropies: total angular momentum method. Phys. Rev. D 56(2), 596-615 (1997a)

26. H. Wayne, M. White, A CMB polarization primer. New Astron. 2(4), 323-344 (1997)
27. M. Kamionkowski, A. Kosowsky, A. Stebbins, Statistics of cosmic microwave background polarization. Phys. Rev. D 55(12), 73687388 (1997)

28. M. Zaldarriaga, U. Seljak, All-sky analysis of polarization in the microwave background. Phys. Rev. D 55(4), 1830-1840 (1997)

29. J. Kim, How to make a clean separation between CMB E and B modes with proper foreground masking. Astron. Astrophys. 531, A32 (2011)

30. D.-H. Kim, S. Trippe, Primordial gravitational waves and rescattered electromagnetic radiation in the cosmic microwave background. Astrophys. J. 830(2), 161 (2016) 